the breast tissue in the axillary tail, with features identical to the core biopsy morphologically and immunologically, and confirmed the diagnosis of a primary breast carcinoma with neuroendocrine differentiation.

The tumour showed a triple negative, basal-like phenotype. Due to the morphology and clinical setting the possibility of a primary elsewhere was considered, however primary lung markers and CK20 were negative, and there was no radiological evidence of other tumours.

Discussion: Male breast cancer though rare remains a morphologically identifiable entity, which can also display rarer morphological patterns such as neuroendocrine differentiation.

\section{DIEULAFOY LESION OF THE GALLBLADDER}

Tarini Fernando, Poh-Yen Yeo

Box Hill Hospital Anatomical Pathology Department, Vic, Australia

We present the case of a 84-year-old female who presented with jaundice, right upper quadrant pain and melaena after a recent anterior resection for diverticular disease. Laboratory investigations showed raised ALP and GGT, low haemoglobin and hyperbilirubinemia. Endoscopy showed fresh bleeding within the third part of the duodenum, with no identifiable source. This triad of upper abdominal pain, jaundice and unknown site of upper gastrointestinal bleed, known as Quincke's triad, is strongly suggestive of haemobilia, and a cholecystectomy was performed. ${ }^{1}$ Macroscopic examination showed a haemorrhagic and acutely inflamed gallbladder. The histological findings demonstrated multi-focal mucosal ulceration with a tortuous medium-sized calibre artery at the base of one of the ulcers, confirming the clinical suspicion of a Dieulafoy lesion.

Dieulafoy lesion is a rare cause of gastrointestinal bleeding, accounting for $1-2 \%$ of all acute emergency digestive bleeds. ${ }^{2}$ The gallbladder is a very rare location for the disease to manifest; the current literature describes only 3 previous cases. The aetiology remains uncertain especially within the gallbladder with multiple hypotheses put forward in the literature. The histological diagnosis requires ample sampling, findings of a larger calibre mucosal or submucosal artery with variable disruption to its integrity protruding through an overlying mucosal defect, and close correlation with the clinical and radiological findings.

\section{References}

1. Chin MW, Enns R, Hemobilia. Curr Gastroenterol Rep 2010; 12: $121-9$.

2. Moszkowicz D, Houdar R. Dieulafoy's lesion of the gallbladder. Surg Radiol Anat 2014; 36: 307-8.

\section{A CASE OF END STAGE SARCOIDOSIS IN AN EXPLANTED HEART}

Tiffany Foo ${ }^{1}$, Peter Dias ${ }^{2}$, Raja Sinniah ${ }^{1}$

${ }^{1}$ Department of Pathology, and ${ }^{2}$ Department of Cardiology, Fiona Stanley Hospital, WA, Australia

Sarcoidosis is a rare granulomatous disease, with the incidence in Australia estimated to be $0.004-0.006 \%$. $^{1}$ A database search of all Pathwest records for the past 20 years yielded only one case of cardiac sarcoidosis. Cardiac sarcoidosis carries a poor prognosis, with a significant proportion developing end-stage cardiac failure, for which heart transplantation is a viable treatment modality. $^{2}$

We present a case of a 63-year-old gentleman who underwent cardiac transplantation for end-stage cardiac sarcoidosis. He presented in 2013 following 2 episodes of syncope. Further investigations with PET, MRI, echocardiogram and CT showed radiological evidence of sarcoidosis. No tissue diagnosis was obtained at the time. He subsequently developed atrial fibrillation with progressive worsening of LV function despite medical treatment. Cardiac transplantation was performed in 2015.

Macroscopic examination showed thickened ventricular walls with multiple patchy, grey, rubbery areas. Microscopic examination showed extensive scarring and fibrosis of the myocardium involving both ventricles and the interventricular septum. Only few poorly formed granulomas were seen despite extensive sampling.

The histopathological diagnosis of sarcoidosis can be challenging due to patchy distribution of granulomas. ${ }^{3}$ Furthermore, there are many mimics which have to be considered. ${ }^{4}$ Careful and widespread sampling is required. ${ }^{4}$

\section{References}

1. Arumugam D, Brown M, Galbraith AM, et al. The incidence of undiagnosed cardiac sarcoidosis in explanted hearts following heart transplantation. Heart Lung Circ 2009; 18S: S1-286.

2. Perkel D, Czer LSC, Morrissey RP, et al. Heart transplantation for end-stage heart failure due to cardiac sarcoidosis. Transplant Proc 2013; 45: 2384-6.

3. Lagana SM, Parwani AV, Nichols LC. Cardiac sarcoidosis: a pathology-focused review. Arch Pathol Lab Med 2010; 134: 1039-46.

4. Bagwan AN, Hooper LVB, Sheppard MN. Cardiac sarcoidosis and sudden death. The heart may look normal or mimic other cardiomyopathies. Virchows Arch 2011; 458: 671-8.

\section{CASE SERIES: TWO CASES OF PRIMARY RETROPERITONEAL MUCINOUS CYSTADENOMA}

Tiffany Foo, Andrew Laycock, Michael Texler

Department of Anatomical Pathology, Fiona Stanley Hospital, WA, Australia

Primary retroperitoneal mucinous cystic tumours are extremely rare. To date, only 56 cases have been reported in the English literature. ${ }^{1,2,3}$ These tumours can be categorised into mucinous cystadenomas, mucinous borderline tumours/tumours of low malignant potential and mucinous carcinomas. ${ }^{4}$ Primary retroperitoneal mucinous cystadenoma (PRMC) accounts for 31 of the reported cases, with the overwhelming majority of cases occurring in females. ${ }^{2,4}$ Due to the rarity of cases, the aetiology and clinical behaviour of this entity is unclear. ${ }^{2}$

In this poster, we present 2 cases of PRMC seen in our department between 2013 and 2015. Both patients were otherwise healthy women who presented with pelvic pain. Further investigations revealed large retroperitoneal cysts with no connections to other intra-abdominal, pelvic or retroperitoneal organs.

Histopathologically, the cysts were lined by bland epithelium alternating between predominantly flattened to cuboidal epithelium, with areas of columnar mucinous epithelial cells. No features of atypia or malignancy were identified. Immunoperoxidase stains showed both the mucinous and flat cuboidal cells to be CK7 positive and CK20 negative. The flat cells were also positive for calretinin, ER and PR while the cytoplasm of the mucinous cells was positive for PAS and PAS-D. 